\title{
Dielectric Interfaces in DC Constructions: Space Charge and Polarization Phenomena
}

\author{
Peter H.F. Morshuis ${ }^{1 *}$, Riccardo Bodega ${ }^{2}$, Davide Fabiani ${ }^{3}$, Gian Carlo Montanari ${ }^{3}$, Len A. Dissado ${ }^{4}$, Johan J. Smit \\ ${ }^{1}$ Delft University of Technology, the Netherlands \\ ${ }^{2}$ Prysmian Cables \& Systems, the Netherlands \\ ${ }^{3}$ University of Bologna, Italy \\ ${ }^{4}$ University of Leicester, United Kingdom \\ * E-mail : p.h.f.morshuis@tudelft.nl
}

\begin{abstract}
Interfaces between dielectrics are considered one of the weakest parts of an insulation system but their behavior under electrical stress is not yet completely understood. In particular, when a dc voltage is applied, the electric field distribution at the interface is quite difficult to predict. This is mainly due to the accumulation of internal charge, which distorts the initial Laplacian field. To shed some new light on this topic, space charge measurements were performed on two types of coaxial XLPE-EPR interfaces.

A numerical procedure, based on the Maxwell-Wagner theory for interfacial polarization, was developed for the estimation of the dynamic charge build-up at the interface. Experimentally obtained space charge profiles were compared to the calculated profiles. The limits of the Maxwell-Wagner model were assessed and the main parameters, which affect the interfacial polarization but which are not included in the model, were identified.
\end{abstract}

\section{INTRODUCTION}

In HV insulation systems inevitably combinations of solid insulating materials are inevitably used. As a consequence, interfaces between dielectrics are very common in $\mathrm{HV}$ components. Typical examples are interfaces in polym eric-type cable accessories (i.e. joints and terminations), between the insulation of the cable and that of the accessory. In case of $\mathrm{HV}$ components working under dc voltage stress conditions, the electric field not only depends on the geometry of the construction and on the permittivity of the insulation, but also on the conductivity of the material and on the presence of space charge. The conductivity of solid insulating materials varies by orders of magnitude with the electric field and temperature, whereas space charge phenomena are very difficult to include in the calculation of the electric field.

In this paper, the electrical behavior of dielectric interfaces is studied on the basis of both experimental investigation and theoretical analysis. Charge profiles were derived from space charge measurements on coaxial XLPE-EPR mini-cables and medium-voltage (MV) size models of cable joints $[1,2]$. A physical model [3] was used for calculating the charge dynamics and electric field distribution in the samples studied, for a range of test conditions.

\section{CHARGE ACCUMULATION AT DIELECTRIC INTERFACES}

The Maxwell-Wagner (MW) theory gives an expression in a closed mathematical form for the calculation of the time dependent surface charge $\kappa(t)$ at the interface between two dielectrics, $\mathrm{A}$ and $\mathrm{B}$. When a de voltage $U_{0}$ is applied across the Maxwell capacitor, the surface charge becomes:

$$
\kappa(t)=\frac{\mathcal{E}_{A} \cdot \sigma_{B}-\varepsilon_{B} \cdot \sigma_{A}}{\sigma_{A} \cdot d_{B}+\sigma_{B} \cdot d_{A}} \cdot U_{0} \cdot\left(1-e^{-\frac{t}{\tau_{M W}}}\right)
$$

where $d_{A}, d_{B}$ are the thickness of the two dielectrics, $\sigma_{A}$, $\sigma_{B}$ the conductivities and $\varepsilon_{A}, \varepsilon_{B}$ the permittivities. The time constant $\tau_{M W}$ is given by

$$
\tau_{M W}=\frac{d_{A} \cdot \varepsilon_{B}+d_{B} \cdot \varepsilon_{A}}{d_{A} \cdot \sigma_{B}+d_{B} \cdot \sigma_{A}}
$$

The main advantage of the MW approach is that the interfacial charge can be directly calculated from the knowledge of a few insulation properties and the value of the insulation thickness. It is to be noted that once the interfacial charge is known, the electric field in both dielectrics can be found:

$$
E_{A(B)}(t)=\frac{U-\frac{\kappa(t) \cdot d_{B(A)}}{\mathcal{E}_{B(A)}}}{d_{A(B)}+d_{B(A)} \cdot \frac{\varepsilon_{A(B)}}{\varepsilon_{B(A)}}}
$$

\section{Modified Maxwell-Wagner approach}

Equation (3) shows that in a combination of two dielectrics the electric field distribution changes in time, because of the accumulation of interfacial charge. The conductivity of insulating materials strongly depends on the electric field and the temperature the material experiences. Thus, also the conductivity changes in time while charges accumulate at the interface. If the field and temperature dependency of the conductivity are taken into account, the conventional MW approach discussed above is no longer valid. Interfacial charge and electric field have to be calculated numerically. In order to consider this phenomenon, the physical model presented in [3] was used. This model is based on the macroscopic properties of the insulation (such as permittivity and conductivity) as a function of the local 
field and temperature. A conductivity function, which is experimentally derived from conduction current measurements, is used as input parameter for the insulation characterization. In this way, a wide range of test conditions can be covered, i.e. different electric fields, temperatures and temperature gradients.

\section{EXPERIMENTAL}

\section{Specimens}

Space charge measurements were performed on two different types of test specimens of increasing complexity, i.e. mini-cables and medium-voltage-size (MV-size) models of cable joints. Figure 1 shows the specimens studied.

Mini-cables [1] consisted of 4-m long triple-extruded constructions. The innermost layer of the mini-cable was made of semicon material, the middle layer of XLPE and the outermost layer of EPR. The total insulation thickness was $2.1 \mathrm{~mm}$ (1.5 mm XLPE +0.6 $\mathrm{mm}$ EPR). An outer semicon was taped on the EPR and a conductive screen was ultimately applied, see Figure 1a. The nature of the interface was chemical, i.e. the interface was cross-linked.

MV-size models of a cable joints [2] were obtained from XLPE-insulated cables (area of the inner conductor $50 \mathrm{~mm}^{2}$; insulation thickness $4.5 \mathrm{~mm}$ ) in which the outer semicon and a part of the XLPE were removed for a length of $80 \mathrm{~mm}$ by means of a glass blade. The insulation was replaced by a $100-\mathrm{mm}$ long elastic tube made of EPR (thickness $2 \mathrm{~mm}$ ). The total insulation thickness of a joint model was $4 \mathrm{~mm} \mathrm{(2 \textrm {mm }}$ $\mathrm{XLPE}+2 \mathrm{~mm}$ EPR). Finally, an outer semicon was taped on the EPR, see Figure 1b-c. The nature of the interface was physical, i.e. the contact was provided by the radial compressive force the EPR cylinder exerts on the XLPE.

One type of HV-quality XLPE, EPR and semicon were used for all test specimens, which were thermally treated prior to any testing in order to expel the volatile by-products of the production process.

\section{Test method and procedure}

Space charge measurements were performed by means of the Pulsed Electroacoustic method (PEA) [4]. The PEA system for coaxial samples described in [1] was adopted for the measurements. Space charge profiles were obtained by processing the acquired raw acoustic signal. Not only deconvolution techniques [5] were used, but also the procedures developed in [6] were adopted for taking into account the fact that the test objects considered in this work are acoustically and electrically heterogeneous.

Measurements were performed at different values of the applied field, in the range $5-20 \mathrm{kV} / \mathrm{mm}$. A temperature drop was applied across the insulation of the test specimens by means of the current-induced heating technique [1].

\section{XLPE-EPR mini-cables}

The development in time of space charge and electric field was calculated for XLPE-EPR mini-cables under the following conditions: $\mathrm{V}=+30 \mathrm{kV} ; \mathrm{T}_{\text {in }}=68^{\circ} \mathrm{C}$; $\mathrm{T}_{\text {out }}=46^{\circ} \mathrm{C} ; \Delta \mathrm{T}=22^{\circ} \mathrm{C} ; \nabla \mathrm{T}=10.5^{\circ} \mathrm{C} / \mathrm{mm}$.

In Figure 2, the results of the calculation are compared to the results of the measurements. The physical model predicts accumulation of charge at the interface between EPR and XLPE because of the discontinuity of permittivity and conductivity. In addition, space charge is predicted in the bulk of the XLPE due to the presence of the temperature gradient. The interfacial charge is slightly underestimated whereas the charge in the XLPE near the inner semicon is slightly overestimated. Nevertheless, the electric field distribution is well predicted by the model.

\section{MV-size models of XLPE-EPR cable joints}

The dynamics of space charge and electric field were calculated for $\mathrm{V}=+80 \mathrm{kV} ; \mathrm{T}_{\mathrm{in}}=65^{\circ} \mathrm{C} ; \mathrm{T}_{\text {out }}=45^{\circ} \mathrm{C}$; $\Delta \mathrm{T}=20^{\circ} \mathrm{C} ; \quad \nabla \mathrm{T}=5{ }^{\circ} \mathrm{C} / \mathrm{mm}$. The results of the calculation and measurements are compared in Figure 3. Initially, experiment and model show negative charge at the interface due to the discontinuity of the permittivity. From a macroscopic point of view, it can be determined that in time positive charge will accumulate at the interface of the cable joint (see Figures $3 a$ and $3 b$ ). The amount of interfacial charge is slightly underestimated by the model. In addition, there is some difference between the charge profiles in the XLPE bulk. The model predicts positive charge near the inner semicon, whereas no charge was measured at that location. As pointed out in [2], there is experimental evidence of accumulation of negative hetero-charge near the inner conductor. The hetero-charge compensates to a large extent the positive charge due to the temperature gradient.

The electric field distribution is rather well predicted by the model. In fact, the calculation indicates that the field increases in the EPR and decreases in the XLPE. Because of the differences observed in the space charge patterns, the maximum value of the field in the EPR near the interface is slightly underestimated by the model (see Figures $3 \mathrm{c}$ and $3 \mathrm{~d}$ ).

\section{Deviation from the MW model}

In general, an acceptable agreement was found between the experimental and calculated patterns. Nevertheless, some of the charging phenomena experimentally observed could not be predicted by the model. In both types of specimens, experimental results showed that space charge is blocked in the XLPE next to the dielectric interface (see dotted circles in Figures $2 \mathrm{~b}$ and $3 b$ ). Because the experiments were performed at an applied electric field above the threshold field for charge injection at the electrode-XLPE interface, we are probably looking at a superimposition of MW charge and injected charge. This injected charge is trapped close to the interface because of the presence of deep physical traps.

Especially in case of a physical interface, such as the 
EPR joint, the interfacial zone can be quite extensive due to the mechanical processing of the materials that compose the interface. Therefore, the nature and distribution of traps near the surface of the insulation is expected to be different from that in the insulation bulk. In another paper presented at this conference [7], a similar behavior was found in minicable specimens consisting of two different dielectrics in physical contact.

The assumption of the presence of deep physical traps and the charge injection at the electrode-XLPE interface may explain the charge blocking phenomenon superimposed on the MW charge in Figures. $2 \mathrm{~b}$ and $3 \mathrm{~b}$.

\section{CONCLUSIONS}

Space charge measurements on dielectric interfaces showed that a model of the interface based on the Maxwell-Wagner theory can predict up to a certain extent the polarization phenomena at the interface.

Therefore the conductivity of the insulation and its dependency on field and temperature play an important role in the charging behavior of dielectric interfaces.

Accumulation of charge near to the interface was not predicted. This was attributed to the microscopic properties of the shallow interface zone of the dielectrics, which are substantially different from those of the insulation bulk. This is especially true for physical interfaces where deep physical traps may lead to charge accumulation. For a proper modeling of the dielectric interface, the differences between surface and bulk of the insulation need to be considered.

\section{Acknowledgement}

This research has been performed in the $5^{\text {th }}$ European Framework Research and Development Program "Benefits of HVDC Links in the European Power Electrical System and Improved HVDC Technology"
(Contract No ENK6-CT-2002-00670).

\section{REFERENCES}

[1] R. Bodega, P.H.F. Morshuis, U.H. Nilsson, G. Perego, J.J Smit, "Charging and polarization phenomena in coaxial XLPE-EPR interfaces", Proc. IEEE Int. Symp. Electr. Insul. Mat., 2005.

[2] R. Bodega, G. Perego, P.H.F. Morshuis, U.H. Nilsson, J.J. Smit, "Space charge and electric field characteristics of polymeric-type MV-size DC cable joint models", Proc. IEEE Conf. on Electr. Insul. Diel. Phenom., pp. 507-510, 2005.

[3] R. Bodega, P.H.F. Morshuis, U.H. Nilsson, G. Perego, J.J Smit, "Polarization mechanisms of flat XLPE-EPR interfaces", Proc. Nord. Ins. Symp, pp.224-227, 2005.

[4] T. Maeno, H. Kushibe, T. Takada, C. M. Cooke, "Pulsed electro-acoustic Method for the measurement of volume charge in e-beam irradiated PMMA", Proc. IEEE Conf. Electr. Insul. Diel. Phenom., pp. 389-397, 1985.

[5] T. Maeno, T. Futami, H. Kushibe, T. Takada, C. M. Cooke, "Measurement of spatial charge distribution in thick dielectrics using the pulsed electroacoustic method", IEEE Trans. Dielectr. Insul., Vol. 23, No. 3, pp 433-439, 1988.

[6] R. Bodega, P.H.F. Morshuis, J.J Smit, "Space charge measurements on multi-dielectrics by means of the pulsed electroacoustic method", IEEE Trans. Dielectr. Electr. Insul., Vol. 13, No. 2, pp.272-281, 2006.

[7] S. Delpino, D. Fabiani, G.C. Montanari, R. Bodega and P.H.F. Morshuis, "The effect of temperature on space charge accumulated at chemical and physical interfaces of HVDC polymeric insulation systems", Proceedings ICSD 2007, Winchester, 2007. a)

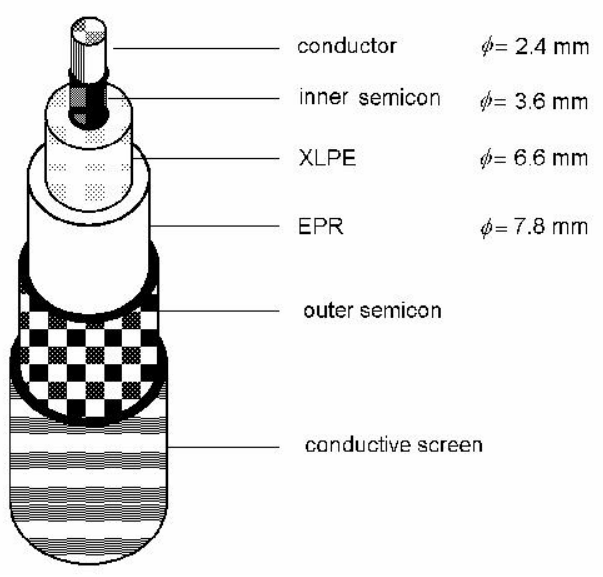

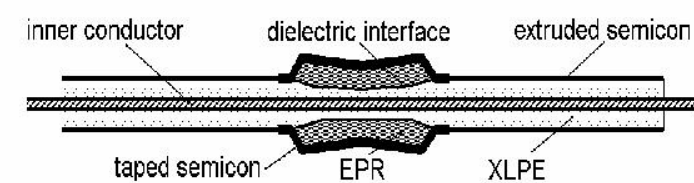

b)

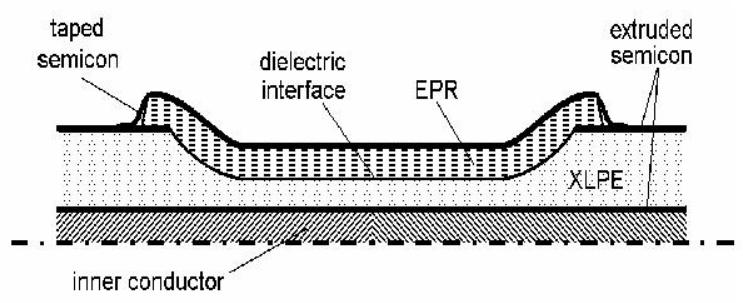

Figure 1: Test specimens in increasing order of complexity: a) mini-cable; b) MV-size model of an EPR joint , c) detail of the interface). 

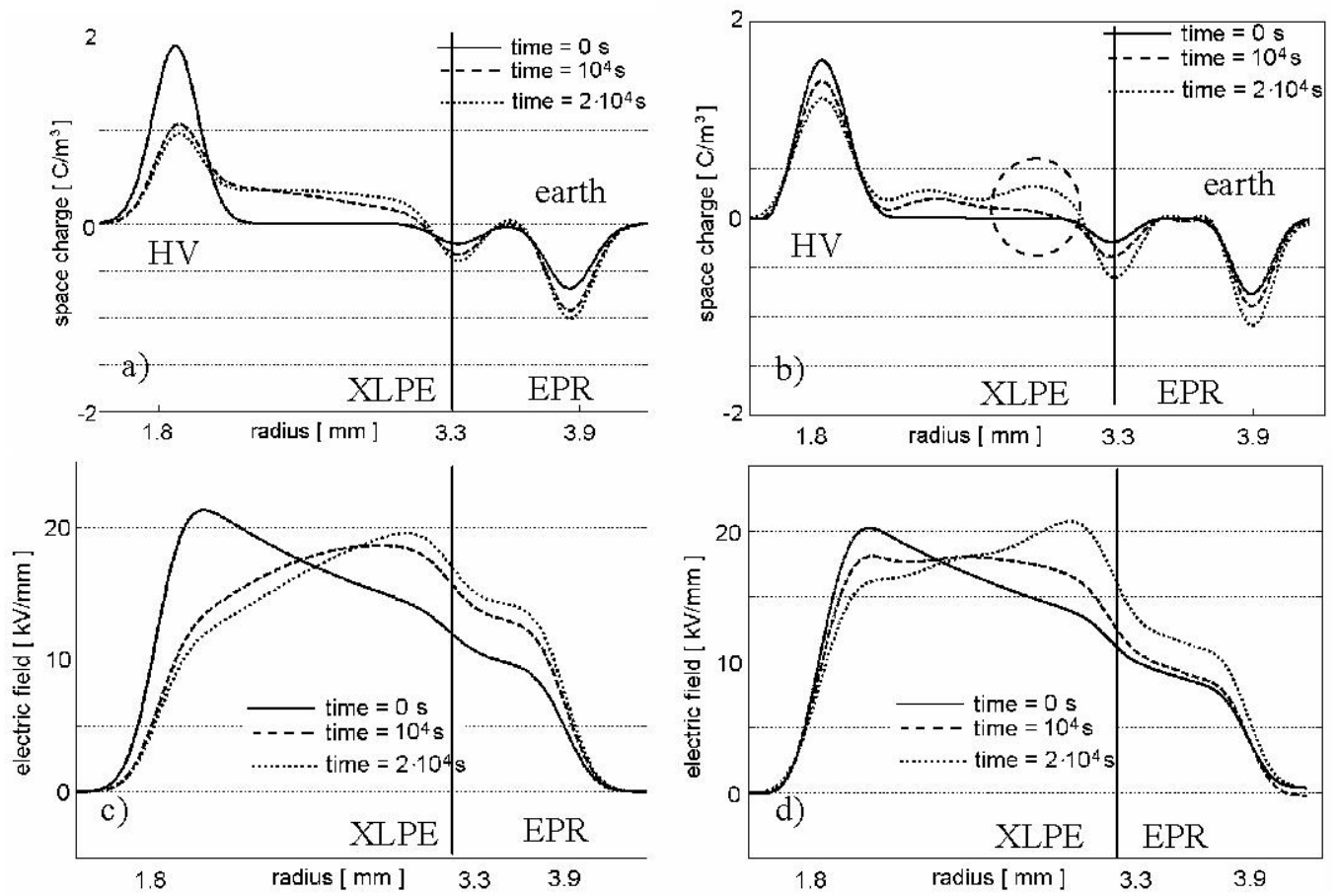

c)

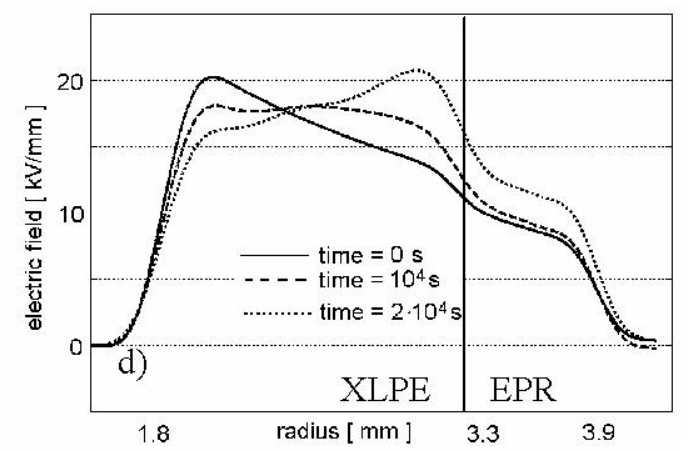

Figure 2: Space charge and electric field profiles of EPR-XLPE mini-cables. $\mathrm{V}=+30 \mathrm{kV} . \mathrm{T}_{\text {in }}=68^{\circ} \mathrm{C} ; \mathrm{T}_{\text {out }}=46^{\circ} \mathrm{C}$; $\Delta \mathrm{T}=22^{\circ} \mathrm{C} ; \nabla \mathrm{T}=10.5^{\circ} \mathrm{C} / \mathrm{mm}$.

a) Calculated charge profiles. b) Measured charge profiles.

c) Calculated electric field profiles. d) Electric field profiles derived from measurements.
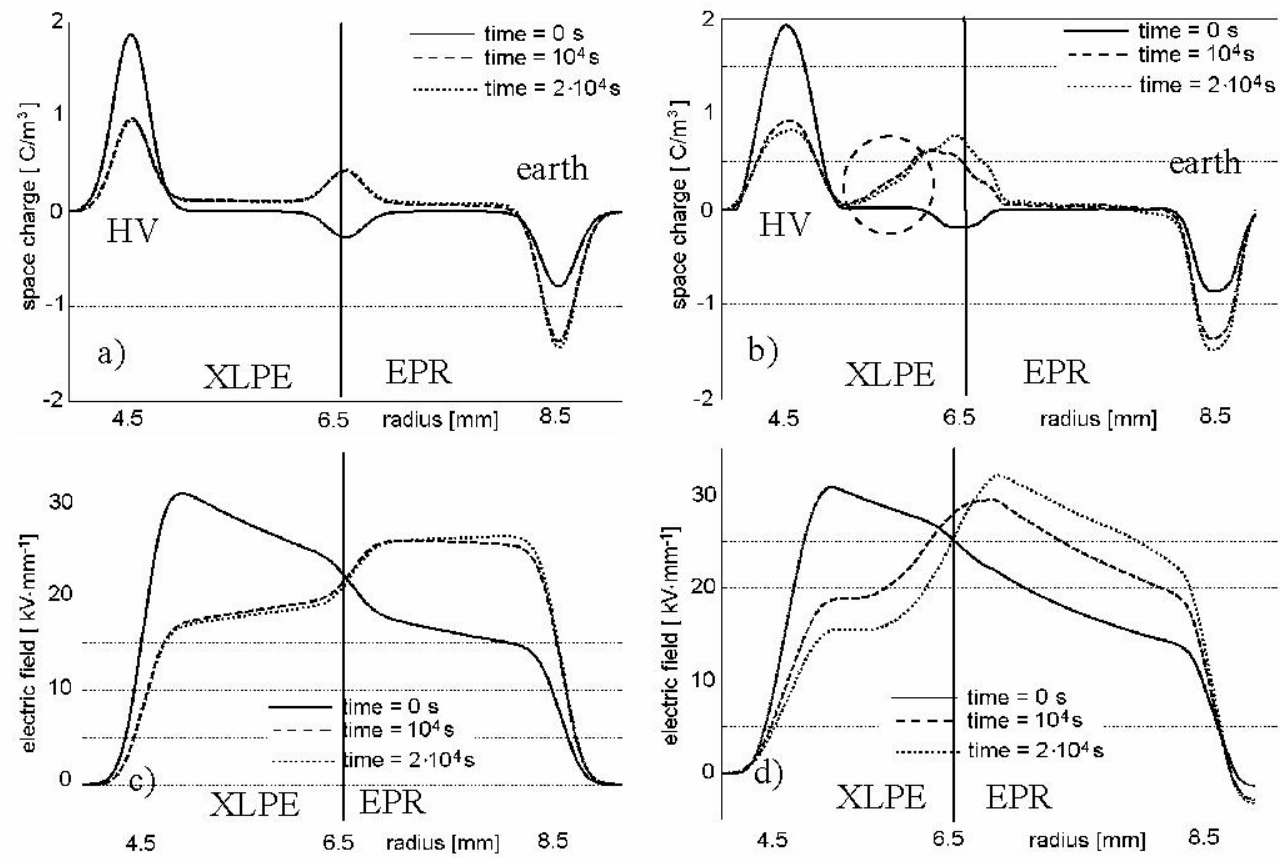

Figure 3: Space charge and electric field profiles of MV-size cable joint models. Applied voltage: $+30 \mathrm{kV}$. $\mathrm{T}_{\text {in }}=65^{\circ} \mathrm{C} ; \mathrm{T}_{\text {out }}=45^{\circ} \mathrm{C} ; \Delta \mathrm{T}=20^{\circ} \mathrm{C} ; \nabla \mathrm{T}=5^{\circ} \mathrm{C} / \mathrm{mm}$.

a) Calculated charge profiles. b) Measured charge profiles.

c) Calculated electric field profiles. d) Electric field profiles derived from measurements. 